\title{
Assessment of costs in open surgery and stereotactic radiosurgery for vestibular schwannomas
}

\author{
Hussam Abou-Al-Shaar, MD, Mohammed A. Azab, MD, Michael Karsy, MD, PhD, MSc, \\ Jian Guan, MD, Gmaan Alzhrani, MD, Yair M. Gozal, MD, PhD, Randy L. Jensen, MD, PhD, and \\ William T. Couldwell, MD, PhD
}

Department of Neurosurgery, Clinical Neurosciences Center, University of Utah, Salt Lake City, Utah

\begin{abstract}
OBJECTIVE Microsurgical resection and radiosurgery remain the most widely used interventions in the treatment of vestibular schwannomas. There is a growing demand for cost-effectiveness analyses to evaluate these two treatment modalities and delineate the factors that drive their total costs. Here, the authors evaluated specific cost drivers for microsurgical and radiosurgical management of vestibular schwannoma by using the Value Driven Outcomes system available at the University of Utah.
\end{abstract}

METHODS The authors retrospectively reviewed all cases involving microsurgical and radiosurgical treatment of vestibular schwannomas at their institution between November 2011 and September 2017. Patient and tumor characteristics, subcategory costs, and potential cost drivers were analyzed.

RESULTS The authors identified 163 vestibular schwannoma cases, including 116 managed microsurgically and 47 addressed with stereotactic radiosurgery (SRS). There were significant differences between the two groups in age, tumor size, and preoperative Koos grade $(p<0.05)$, suggesting that indications for treatment were markedly different. Length of stay (LOS) and length of follow-up were also significantly different. Facility costs were the most significant contributor to both microsurgical and SRS groups (58.3\% and $99.4 \%$, respectively); however, physician professional fees were not specifically analyzed. As expected, microsurgical treatment resulted in an average 4 -fold greater overall cost of treatment than for SRS cases $(p<0.05)$, and there was a greater variation in costs for open cases as well. Costs remained stable over time for both open resection and SRS. Multivariable analysis showed that LOS $(\beta=0.7, p=0.0001)$, discharge disposition ( $\beta=0.2, p=0.004)$, nonserviceable hearing $(\beta=0.1, p=0.02)$, and complications $(\beta=0.2, p=0.005)$ affected cost for open surgery, whereas no specifically examined factor could be identified as driving costs for SRS.

CONCLUSIONS This analysis identified the fact that facility utilization constitutes the majority of total costs for both microsurgery and SRS treatment modalities of vestibular schwannomas. LOS, discharge disposition, nonserviceable hearing, and complications contributed significantly to the total costs for the microsurgical group, whereas none of the factors could be identified as driving total costs for the SRS group. This information may be used to establish policies and protocols to reduce facility costs, with the goal of decreasing the total costs without jeopardizing patient care.

https://thejns.org/doi/abs/10.3171/2018.4.JNS18365

KEYWORDS cost-effectiveness; vestibular schwannomas; retrosigmoid; translabyrinthine; middle cranial fossa; stereotactic radiosurgery; value-driven outcome

$\mathrm{V}$ ESTIBULAR schwannomas are relatively uncommon skull base tumors that arise from the vestibular branch of the eighth cranial nerve and account for up to $10 \%$ of all intracranial neoplasms. ${ }^{11}$ The incidence of vestibular schwannomas has spiked dramatically in re- cent years, from 0.3 cases per 100,000 in 1976 to 1.9 cases per 100,000 in 2008 as more tumors have been identified because of the increased use of MRI. ${ }^{15}$ Management options include conservative observation, radiosurgery, and microsurgical resection. ${ }^{21}$ Various patient-level factors are

ABBREVIATIONS ASA = American Society of Anesthesiologists; GKS = Gamma Knife radiosurgery; LOS = length of stay; $\mathrm{mRS}=$ modified Rankin Scale; SRS = stereotactic radiosurgery; VDO = Value Driven Outcomes.

SUBMITTED February 7, 2018. ACCEPTED April 9, 2018

INCLUDE WHEN CITING Published online October 5, 2018; DOI: 10.3171/2018.4.JNS18365. 
analyzed when choosing the appropriate treatment modality, including the patient's age, physical status, tumor size, tumor growth rate, and degree of functional hearing, among other factors. ${ }^{8,9}$ Therefore, the preference for one technique over another remains controversial. Recently, the rise of healthcare costs has led to greater pressure to identify ways to reduce the costs of care regardless of treatment modality.

Microsurgical resection and radiosurgery are the most widely used interventions in the treatment of vestibular schwannomas, and several studies have compared the costs of the two treatments. Although it is typically shown that radiosurgery is less expensive than open resection, $1,2,18-20,23$ most studies comparing the costs between the two modalities have not considered the subcategory costs (e.g., facility, imaging, pharmacy) that drive total costs. In an effort to identify these factors, we used direct costs, rather than charge- or revenue-based tools, to analyze costeffectiveness. We have previously used the Value Driven Outcomes (VDO) tool at the University of Utah for analyses of cost drivers; by identifying these most costly components of patient care, our goal is to uncover new ways to reduce cost expenditure while maintaining a high quality of care. ${ }^{4,5}$ Our objective here was to evaluate specific cost drivers for microsurgical and radiosurgical management of vestibular schwannomas by using the VDO system.

\section{Methods}

\section{Patient Inclusion}

The institutional review board granted approval of this study with a waiver of informed consent. We used the VDO database and a cross-referenced electronic medical record to identify patient cases with microsurgical resection or stereotactic radiosurgery (SRS) of vestibular schwannoma treated between November 2011 and September 2017. Patients with open microsurgical resection of vestibular schwannomas were identified from an internal database maintained by the senior author (W.T.C.). Patients who underwent SRS were identified by crossreferencing Current Procedural Terminology (CPT) codes for SRS (e.g., 61796, 61798, 77371, 77372, 77373, 77432, and 77435) with an ICD-10 code for benign neoplasm of cranial nerves (i.e., D33.3). Screening of patients who underwent microsurgical resection of vestibular schwannomas by CPT or ICD-10 code alone did not yield patients because of billing code changes over time. Patients were excluded if they were $<18$ years of age, did not have an elective procedure for the treatment of vestibular schwannoma, or had previous treatment for a vestibular schwannoma. Patients were also excluded if they lacked complete clinical, radiographic, and cost data. A manual chart review was performed to verify patients' inclusion and to obtain clinical variables.

\section{Surgical Procedures}

Patients who underwent open surgery had retrosigmoid, translabyrinthine, or middle cranial fossa approaches depending on specific patient details such as tumor size, location, and patient hearing function. All open surgical cases were performed by the senior author. SRS involved a single-dose fraction (14 Gy) that was intended to reduce the dosage to the cochlea and brainstem. All SRS cases were performed by a neurosurgeon (R.L.J.) in combination with the radiation oncology team. Patients who underwent open surgery and developed a CSF leak underwent conservative therapy, including observation, oversuturing, and lumbar drain placement. Severe cases of CSF leakage that did not recover with conservative treatment were managed surgically. All cases of complications, whether medically or surgically managed, were captured by cost analysis because they occurred during the same patient encounter.

\section{Data Analysis}

We obtained demographic and surgical data from the medical records for each patient. Demographic data included patient age and sex. Patient status before surgery was assessed with the American Society of Anesthesiologists (ASA) physical status system. Tumor size, hearing function, Koos grade, and modified Rankin Scale (mRS) score were also identified. Nonserviceable hearing was defined as a patient having a pure tone average of $<50$ $\mathrm{dB}$ and speech discrimination tone $<50 \%$. Complications were classified as minor (complications managed conservatively) or major (complications requiring surgical intervention or potentially threatening the patient's life). Length of stay (LOS) and discharge disposition were captured as well.

The VDO database is an electronic resource that reports direct costs, in lieu of patient/insurer charges, and its methodology has been previously reported. ${ }^{4,5}$ Total cost and subcategory costs, including pharmacy, imaging, supplies and implants, laboratory, and facility costs, were analyzed. Physician professional fee was not available as a subcategory. Actual dollar amounts are not reported, per agreement with the university. Subcategory cost is reported as a percentage of total cost. The mean percentage of total cost was generated as an alternative to presenting actual cost data. Costs were totaled for the entire cohort of patients, and the fraction of total cost contribution for each patient was calculated. Thus, means, standard deviations, patient total, and subgroup costs could be compared. The mean percentage of total costs may not necessarily total $100 \%$. For subgroup cost contribution, each patient's subgroup cost was divided by the total. For these calculations, percentages will equal $100 \%$.

Continuous variables are reported as means and standard deviations and were analyzed by t-test. Noncontinuous variables were analyzed by chi-square test. A multivariable linear regression was used to correlate factors with cost. Variables that showed a $p$ value $<0.2$ on univariable analysis were included in the multivariable analysis. Variables were evaluated for multicollinearity by using tolerance and variance inflation factor cutoffs of $<0.2$ and $>$ 5 , respectively. In lieu of reporting unstandardized beta values in the regression analysis to demonstrate how the variance of factors affects cost, we have used the standardized beta, which allows comparability of variable magnitude and directions. SPSS software (V20.0; IBM) was used for statistical analysis, with $\mathrm{p}<0.05$ considered significant. 


\section{Results}

\section{Patient Demographics, Surgical Characteristics, and Procedures}

Of the 163 patients with vestibular schwannoma treated during the study period, 116 were treated microsurgically and 47 were treated with SRS (Table 1). There was a significant difference in age between the two groups, with a mean age in the surgical group of $49.7 \pm 12.8$ years and a mean age in the SRS group of $68.5 \pm 12.3$ years $(p=$ $0.0001)$. No significant difference in sex was seen between the surgical $($ male $=45.7 \%)$ and SRS $(48.9 \%)$ groups $(p=$ 0.6 ). There was a statistically significant difference in the length of the follow-up periods between the microsurgical and SRS groups $(17.2 \pm 25.3$ vs $20.9 \pm 16.9$ months, respectively) $(\mathrm{p}=0.0001)$.

The patients in the surgical group had a mean hospital LOS of $5.1 \pm 3.5$ days, whereas those in the SRS group, who were treated as outpatients, had a mean LOS of 1 day $(p=0.001)$. The most common fiscal year for the surgical and SRS groups was 2016 and 2015, respectively, suggesting that the timing for treatment of patients in both groups was similar. No statistically significant difference in the rate of discharge to home/self-care was noted between the two groups $(78.4 \%$ vs $100 \%)(p=0.1)$. Additionally, the mRS did not differ significantly, with the majority of patients having no significant disability $(\mathrm{mRS} \geq 2)$ after their treatment $(94.9 \%$ vs $87.3 \%)(p=0.2)$. The ASA classification for open surgery was healthy $(\mathrm{n}=12,10.3 \%)$, mild ( $\mathrm{n}$ $=63,54.3 \%)$, severe $(\mathrm{n}=23,19.8 \%)$, incapacitating $(\mathrm{n}=1$, $1.0 \%$ ), and missing $(\mathrm{n}=17,14.7 \%)$.

There was a statistically significant difference in tumor size between the surgical and SRS groups $(2.2 \pm 1.4$ vs 1.6 $\pm 0.7 \mathrm{~cm}$, respectively) $(\mathrm{p}=0.0001)$. Nonserviceable hearing was present in $14.7 \%$ of the surgical group and in $10.6 \%$ of the SRS group before treatment. Minor complications occurred in $10.3 \%$ of the surgical group, with CSF leakage being the most commonly encountered minor complication and sinus thrombosis being the most common major complication (Table 1). No complications were identified within the SRS group. The Koos grade was significantly different between the surgical and SRS groups $(\mathrm{p}=0.004)$. The fundal fluid cap was found in similar numbers of patients in the two groups $(57.8 \%$ vs $68.1 \%)(\mathrm{p}=0.2)$.

\section{Cost Subgroup Analysis}

Subcategory cost drivers were evaluated for each treatment modality (Fig. 1). For open microsurgical resection, facility costs made the largest $(58.3 \%)$ contribution to total costs, followed by supplies (25.6\%), pharmacy (13.1\%), laboratory (2.3\%), and imaging $(0.6 \%)$. On the other hand, for SRS, facility costs were the only significant cost driver (99.4\%); supplies, pharmacy, imaging, and laboratory costs collectively contributed to only $0.6 \%$ of the total costs. When evaluating the subcategory and total costs, there was a statistically significant difference for all subcategory costs and the total cost between treatment types ( $\mathrm{p}<0.05$, t-test).

On average, open surgery cost approximately 4-fold more than radiosurgery per case (Fig. 1C and D). Variability in subtotal costs for different treatment types is shown in Fig. 1D. Between 2011 and 2017, there was significant difference in cost variability between the surgical and SRS groups, with greater variation among open surgical ( $\beta=0.072, p=0.47$; linear regression) than SRS cases, as is apparent by the distribution of the scatterplot points (Fig. 1E). Overall, costs remained stable for both open resection $(\beta=0.072, p=0.47$; linear regression) and SRS $(\beta$ $=-0.001, \mathrm{p}=0.99$; linear regression).

Univariable and multivariable linear regression were used to assess cost drivers for each group (Tables 2 and $3)$. For open microsurgical cases, multivariable linear regression showed that $\operatorname{LOS}(\beta=0.7, p=0.0001)$, discharge disposition $(\beta=0.2, p=0.004)$, nonserviceable hearing $(\beta=0.1, p=0.02)$, and complications $(\beta=0.2, p=0.005)$ affected total costs. Notably, LOS played the most significant role, as evidenced by the largest $\beta$ value. For SRS cases, no variable was identified on multivariable analysis that had a significant effect on total cost.

\section{Discussion}

\section{Study Findings}

In this study, we have shed light on key clinical factors and cost drivers that contribute to the total costs for treating patients with microsurgery or SRS for vestibular schwannoma. As reported in other studies, ${ }^{1,2,18,19,23}$ the total costs for SRS were less than those of open microsurgical resection. Our results showed a 4-fold difference in total cost; however, the primary objective of this study was to delineate the subcategory costs and cost drivers that contribute to the total cost difference between the two groups, because these subcategory costs are rarely reported in the literature. The overall differences in cost distributions showed that facility costs were predominant in both surgical and SRS groups, but that the degree of facility contribution to the total cost was statistically different between surgical and SRS treatments $(58.3 \%$ vs $99.4 \%)(\mathrm{p}=$ 0.04 , t-test). There was also greater variation in costs for microsurgically resected cases compared with SRS cases. However, costs remained stable over time for both open resection and SRS.

Facility costs were the predominant contributor to cost for the treatment of vestibular schwannomas by either open surgery or radiosurgery. Our previous work illustrated the same finding in regard to sellar tumors and pituitary adenomas. ${ }^{4,5,10,16,17}$ As previously reported, ${ }^{6}$ facility costs mostly encompass the direct salaries of hospital staff (e.g., nurses and technicians) and costs to maintain the hospital (including equipment depreciation), but also include salaries for support staff (e.g., administrators and maintenance workers), insurance, and other ancillary costs. This amount is directly correlated to the billed facility charges and is separate from bills for professional services. Future work will need to evaluate where costs can be reduced in this domain for various specialties and treatments.

The clinical factors that had the most influence on costs for open surgical procedures in this study were LOS, discharge disposition, nonserviceable hearing, and complications, whereas no clinical factor could be identified on multivariable analysis as having a significant role in determining costs. These factors were identified by analysis 
TABLE 1. Baseline demographic and surgical variables for open surgery and SRS treatment of vestibular schwannomas

\begin{tabular}{|c|c|c|c|c|}
\hline Variable & All Patients, $N=163$ & Open Surgery, $n=116$ & Radiosurgery, $n=47$ & $\mathrm{p}$ Value \\
\hline Age in yrs & $55.7 \pm 15.4$ & $49.7 \pm 12.8$ & $68.5 \pm 12.3$ & 0.0001 \\
\hline Male sex & $76(46.6 \%)$ & $53(45.7 \%)$ & $23(48.9 \%)$ & 0.6 \\
\hline ASA class & & & & 0.0001 \\
\hline Healthy & $12(7.4 \%)$ & $12(10.3 \%)$ & & \\
\hline Mild disease & $63(38.7 \%)$ & $63(54.3 \%)$ & & \\
\hline Severe disease & $23(14.1 \%)$ & $23(19.8 \%)$ & & \\
\hline Incapacitating disease & $1(0.6 \%)$ & $1(1.0 \%)$ & & \\
\hline Unknown & $64(39.3 \%)$ & $17(14.7 \%)$ & $47(100.0 \%)$ & \\
\hline LOS in days (IQR) & $4.3 \pm 5.7(1-5)$ & $5.1 \pm 3.5(3-5)$ & 1 & 0.001 \\
\hline Neuro-ICU LOS in days & $2.0 \pm 2.4$ & $2.0 \pm 2.4$ & 0 & NA \\
\hline Neuro-floor LOS in days & $2.8 \pm 2.2$ & $2.8 \pm 2.2$ & 0 & NA \\
\hline Discharge disposition & & & & 0.1 \\
\hline Home/self-care & $138(84.7 \%)$ & $91(78.4 \%)$ & $47(100.0 \%)$ & \\
\hline Skilled nursing facility & $2(1.2 \%)$ & $2(1.7 \%)$ & $0(0.0 \%)$ & \\
\hline Acute rehab facility & $7(4.3 \%)$ & $7(6.0 \%)$ & $0(0.0 \%)$ & \\
\hline Unknown & $16(9.8 \%)$ & $16(13.8 \%)$ & $0(0.0 \%)$ & \\
\hline 1-yr mRS outcomes & & & & 0.2 \\
\hline 0, no symptoms & $3(1.8 \%)$ & $1(1.0 \%)$ & $2(4.3 \%)$ & \\
\hline 1 , no significant disability & $138(84.7 \%)$ & $102(87.9 \%)$ & $36(76.6 \%)$ & \\
\hline 2, slight disability & $10(6.1 \%)$ & $7(6.0 \%)$ & $3(6.4 \%)$ & \\
\hline 3 , moderate disability & $7(4.3 \%)$ & $3(2.6 \%)$ & $4(8.5 \%)$ & \\
\hline 4 , moderately severe disability & $4(2.5 \%)$ & $3(2.6 \%)$ & $1(2.1 \%)$ & \\
\hline 5 , severe disability & $0(0.0 \%)$ & $0(0.0 \%)$ & $0(0.0 \%)$ & \\
\hline 6, dead & $0(0.0 \%)$ & $0(0.0 \%)$ & $0(0.0 \%)$ & \\
\hline Unknown & $1(0.6 \%)$ & $0(0.0 \%)$ & $1(2.1 \%)$ & \\
\hline Follow-up in mos & $18.3 \pm 23.2$ & $17.2 \pm 25.3$ & $20.9 \pm 16.9$ & 0.0001 \\
\hline Max tumor size in $\mathrm{cm}$ & $2.0 \pm 1.3$ & $2.2 \pm 1.4$ & $1.6 \pm 0.7$ & 0.0001 \\
\hline Nonserviceable hearing & $22(13.5 \%)$ & $17(14.7 \%)$ & $5(10.6 \%)$ & 0.5 \\
\hline Complications & & & & NA \\
\hline Minor & & $12(10.3 \%)$ & & \\
\hline CSF leak w/ conservative management & & $8(6.9 \%)$ & & \\
\hline Abdominal incision hematoma & & $1(0.8 \%)$ & & \\
\hline Aseptic meningitis & & $1(0.8 \%)$ & & \\
\hline Tympanic membrane injury & & $1(0.8 \%)$ & & \\
\hline Superficial wound infection & & $1(0.8 \%)$ & & \\
\hline Major & & $13(11.2 \%)$ & & \\
\hline CSF leak w/ eustachian tube closure & & $1(0.8 \%)$ & & \\
\hline CSF leak w/ wound closure & & $1(0.8 \%)$ & & \\
\hline Transverse sinus thrombosis & & $6(5.2 \%)$ & & \\
\hline Postop hemorrhage & & $2(1.7 \%)$ & & \\
\hline Meningitis \& abscess & & $1(0.8 \%)$ & & \\
\hline Wound infection w/ bone flap removal & & $1(0.8 \%)$ & & \\
\hline Hydrocephalus w/ VP shunt & & $1(0.8 \%)$ & & \\
\hline Koos grade & & & & 0.004 \\
\hline 1, intracanalicular & $54(33.1 \%)$ & $34(29.3 \%)$ & $20(42.6 \%)$ & \\
\hline $2, \leq 2 \mathrm{~cm}$ & $54(33.1 \%)$ & $33(28.4 \%)$ & $21(44.7 \%)$ & \\
\hline $3, \leq 3 \mathrm{~cm}$ & $14(8.6 \%)$ & $11(9.5 \%)$ & $3(6.4 \%)$ & \\
\hline $4,>3 \mathrm{~cm}$ & $38(23.3 \%)$ & $36(31.0 \%)$ & $2(4.3 \%)$ & \\
\hline Missing & $3(1.8 \%)$ & $2(1.7 \%)$ & $1(2.1 \%)$ & \\
\hline Fundal fluid cap & $99(60.7 \%)$ & $67(57.8 \%)$ & $32(68.1 \%)$ & 0.2 \\
\hline
\end{tabular}


$\mathrm{IQR}=$ interquartile range; $\max =$ maximum; $\mathrm{NA}=$ not applicable; $\mathrm{VP}=$ ventriculoperitoneal.

Continuous values are presented as the mean \pm SD. Unless otherwise indicated, other data are presented as the number of patients (\%). Boldface type indicates statistical significance.

of our cohort of 163 patients with vestibular schwannoma who underwent treatment by open microsurgical resection $(n=116)$ or SRS $(n=47)$. These two groups differed in several ways. Age was significantly different between the two groups ( $\mathrm{p}=0.0001)$, with younger patients in the surgical group. The observed difference could be explained by the preference of young patients to undergo microsurgical resection to avoid the potential of tumor growth, risk of malignant transformation, and/or long-term adverse effects of SRS. ${ }^{37,12}$ Failed radiosurgery in younger patients often mandates resection as a salvage therapy later in life, which can impact surgical complexity, potential complications, and overall treatment costs. ${ }^{22}$ Moreover, tumor size was significantly larger in open surgical cases compared with the SRS group $(2.2 \pm 1.4$ vs $1.6 \pm 0.7 \mathrm{~cm})(\mathrm{p}=0.0001)$. Tumors larger than $2-3 \mathrm{~cm}$ are often more likely to undergo microsurgical resection compared with smaller tumors, which can be managed conservatively or with radiosurgery, because large tumors have the potential to produce mass effect and show radioresistance. The Koos grade was also different between the two groups $(\mathrm{p}=0.004)$. Interestingly, no statistically significant difference in 1-year mRS outcomes was encountered between the open surgical and SRS groups.

\section{Vestibular Schwannoma Treatment Cost and Cost- Containment Strategies}

A number of previous studies have sought to examine the cost of vestibular schwannoma treatment. It is well established that SRS is less expensive than open microsurgical resection of vestibular schwannoma. Banerjee et al. ${ }^{1}$ reported a mean total cost of $\$ 23,788$ (95\% CI $\$ 22,280-\$ 24,842)$ for microsurgically treated patients who were followed for $\geq 36$ months, whereas the mean costs for patients who underwent SRS were \$16,143 $(95 \%$ CI $\$ 15,277-\$ 17,545)$. Interestingly, the authors found that although the total cost of microsurgery was higher than the total cost of SRS, the follow-up costs were higher in the SRS group. The primary reason for higher follow-up costs after SRS was the greater frequency of imaging and hearing evaluations in those patients compared with the surgical group. The authors concluded that as long as the recurrence rate of vestibular schwannoma remains low, SRS remains a more cost-effective modality. We did not evaluate the follow-up imaging costs for patients who underwent microsurgical or SRS treatments. The effect of numerous additional clinical follow-up encounters and imaging studies could be added to our findings and might affect our results.

Similarly, Caruso and colleagues ${ }^{2}$ reported that the average 12-month costs of care for treating vestibular schwannoma microsurgically or with Gamma Knife radiosurgery (GKS) were $\$ 67,538$ and $\$ 29,698$, respectively.
In a European study ${ }^{18}$ that focused on the direct (procedural and hospital-related) and indirect costs (missed work days) of vestibular schwannoma management, van Roijen et al. found that radiosurgery had lower direct and indirect costs compared with open surgery, whereas differences in clinical outcomes between groups were insignificant. Interestingly, Verma et al. ${ }^{19}$ reported that treatment with GKS for small tumors was more expensive in Canada (CAD27,659) than open microsurgical resection $(\mathrm{CAD} 22,402)$ for treatment of vestibular schwannoma. In this study all patients were initially assigned to conservative management, and those who failed proceeded with treatment. However, all treatments with GKS were performed in the US, because of the lack of GKS facilities in Canada during this time frame. This study also helped highlight the significant geographic variations in cost even for treatment of the same disease.

Slattery et al. ${ }^{13}$ studied the association between center volume and hospital costs. Using a hospital discharge database in California to evaluate cases treated between 1996 and 1998, the authors concluded that the total charges and costs per day decrease as the center volume increases $(\mathrm{p}<$ 0.001 ). We expect that this is because higher case volume probably improves patient outcomes and reduces complications, which ultimately reduces cost. Interestingly, Sonig et al. ${ }^{14}$ analyzed the Nationwide Inpatient Sample, a database of approximately $20 \%$ of hospital discharges in the US, to understand the socioeconomics of vestibular schwannoma surgery. The average charge per patient was $\$ 76,365.09 \pm \$ 58,039.93$. The authors correlated increased cost with larger hospitals, teaching institutions, the Western region, adverse inpatient events, and comorbidities. The advantage of our data is that they include actual cost data during the treatment of vestibular schwannoma, rather than patient charges, which adds to our current understanding of cost in the management of this disease.

Among the reports in the literature comparing the costs of open surgery and radiosurgery for vestibular schwannoma management, only a minority have examined the subgroup analyses and cost drivers. In a German study, Wellis et al. ${ }^{20}$ reported the average overall costs for primary microsurgical therapy were $€ 10,814 \pm € 6,108$, with subgroup costs of $€ 1,417 \pm € 426$ for the surgical procedure; $€ 1,188 \pm € 2,658$ for intensive care unit stay; $€ 2,333$ $\pm € 1,582$ for medical and nursing care on the ward; $€ 1,671 \pm € 1,433$ for interclinical bills (i.e., bills from other departments); and $€ 4,204 \pm € 2,338$ for basic overhead (€273/day). However, their study included patients with vestibular schwannomas, meningiomas, brain metastases, and arteriovenous malformations, and it did not specify the costs for the vestibular schwannoma group alone. Zygourakis et al. ${ }^{23}$ looked at the differences in cost drivers between vestibular schwannoma treatment modalities. 

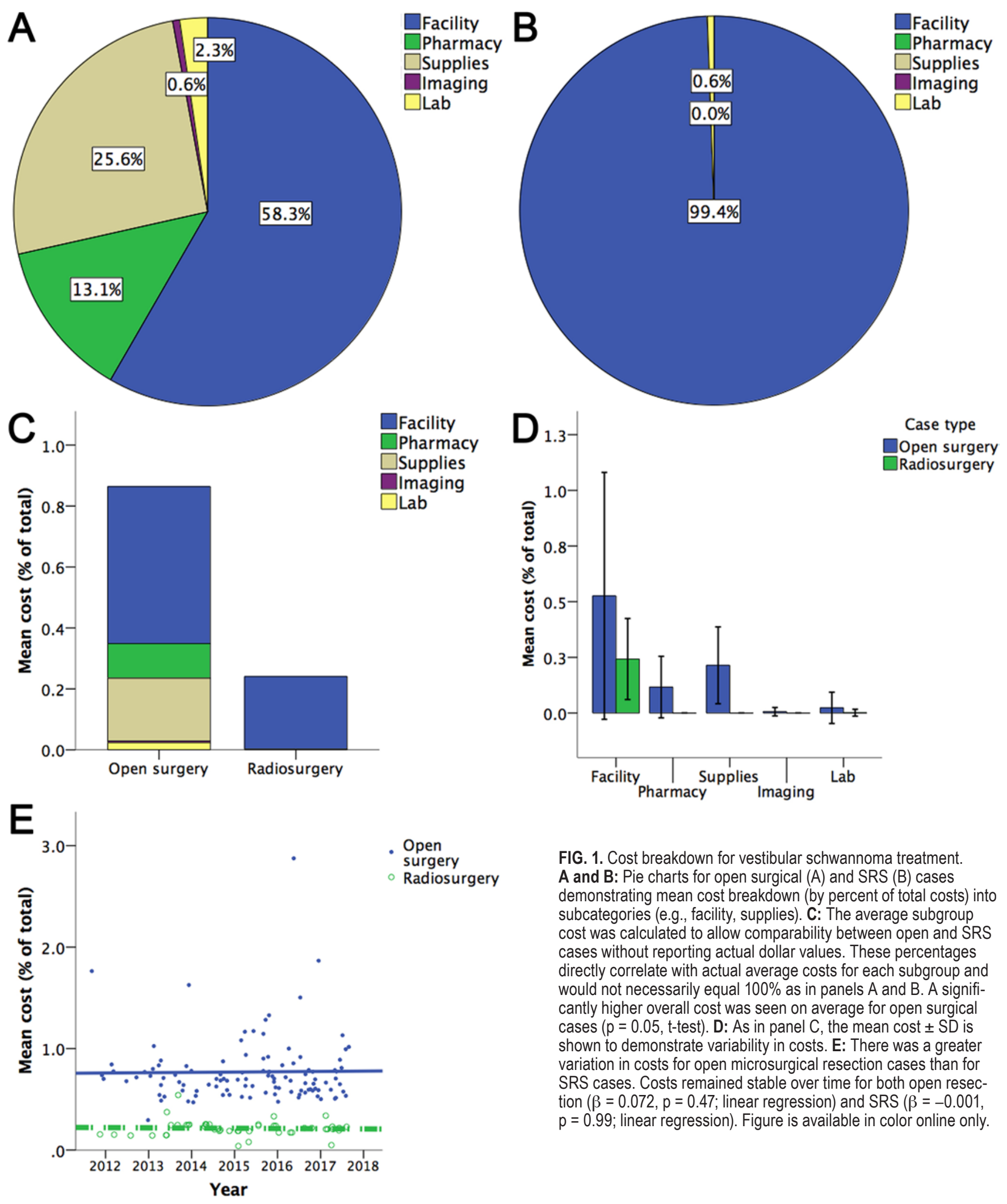

Case type

Open surgery Radiosurgery

FIG. 1. Cost breakdown for vestibular schwannoma treatment. $A$ and B: Pie charts for open surgical (A) and SRS (B) cases demonstrating mean cost breakdown (by percent of total costs) into subcategories (e.g., facility, supplies). C: The average subgroup cost was calculated to allow comparability between open and SRS cases without reporting actual dollar values. These percentages directly correlate with actual average costs for each subgroup and would not necessarily equal $100 \%$ as in panels A and B. A significantly higher overall cost was seen on average for open surgical cases $(p=0.05$, t-test). $D$ : As in panel $C$, the mean cost $\pm S D$ is shown to demonstrate variability in costs. $\mathrm{E}$ : There was a greater variation in costs for open microsurgical resection cases than for SRS cases. Costs remained stable over time for both open resection ( $\beta=0.072, p=0.47$; linear regression) and SRS $(\beta=-0.001$, $p=0.99$; linear regression). Figure is available in color online only.

They found that the total costs for patients in the surgery group were $\$ 80,074 \pm \$ 49,678$, compared with $\$ 9,737 \pm$ $\$ 5,522$ for patients receiving GKS. They found that the op-

erating room and hospital room costs represented $43.3 \%$ and $44.6 \%$ of total inpatient costs, respectively, for patients in the surgery group, whereas imaging costs represented 
TABLE 2. Univariable and multivariable analysis of cost drivers for open surgery

\begin{tabular}{|c|c|c|c|c|}
\hline \multirow[b]{2}{*}{ Variable } & \multicolumn{2}{|c|}{ Univariable } & \multicolumn{2}{|c|}{ Multivariable } \\
\hline & $\begin{array}{c}\text { Standardized } \\
\beta\end{array}$ & $\begin{array}{c}p \\
\text { Value }\end{array}$ & $\begin{array}{c}\text { Standardized } \\
\beta\end{array}$ & $\begin{array}{c}p \\
\text { Value }\end{array}$ \\
\hline Age in yrs & -0.04 & 0.7 & & \\
\hline Male sex & -0.07 & 0.5 & & \\
\hline ASA class & 0.1 & 0.2 & 0.01 & 0.8 \\
\hline LOS in days & 0.9 & 0.0001 & 0.7 & 0.0001 \\
\hline $\begin{array}{l}\text { Discharge } \\
\text { disposition }\end{array}$ & 0.5 & 0.0001 & 0.2 & 0.004 \\
\hline $\begin{array}{l}\text { Max tumor size } \\
\text { in } \mathrm{cm}\end{array}$ & 0.3 & 0.001 & -0.07 & 0.4 \\
\hline $\begin{array}{l}\text { Nonserviceable } \\
\text { hearing }\end{array}$ & 0.3 & 0.01 & 0.1 & 0.02 \\
\hline Complications & 0.5 & 0.0001 & 0.2 & 0.005 \\
\hline Koos grade & 0.3 & 0.001 & 0.1 & 0.07 \\
\hline Fundal fluid cap & -0.1 & 0.3 & & \\
\hline
\end{tabular}

Boldface type indicates statistical significance.

$45.2 \%$ of the total outpatient costs. On the other hand, the majority $(80.5 \%)$ of costs for their radiation group were from GKS costs, whereas imaging costs represented only $11 \%$ of the total cost. The authors concluded that surgery is a cost-effective alternative to radiation in patients aged $<45$ years, whereas for patients $\geq 45$ years old, radiation is the most cost-effective treatment modality.

Our report suggests that microsurgical resection generates a significant upfront cost compared with SRS. Although follow-up imaging after SRS was not examined in our study, it is unlikely that imaging studies would have eliminated or noticeably narrowed the 4-fold difference in cost between resection and SRS, assuming that retreatment was not required. Whereas Zygourakis et al. performed a decision-tree analysis using published quality of life metrics and estimates of treatment cost, the advantage of the VDO is in actual subgroup analysis and identification of specific cost drivers, which adds greatly to the growing body of literature on this topic. Thus, by comparing total direct cost, one can better identify unexpected subgroup costs and contributing clinical factors. One potential next step would be to determine the difference in quality of life and serviceable hearing outcomes by using cost analysis with the VDO.

\section{Limitations of the Study}

Several limitations of our study include its retrospective nature, single-site perspective, and unequal small sample size between the two groups. In this retrospective study, we were limited to the data recorded in the patient records and the VDO database. In addition, the management, training, and facilities at our institution may differ from those at other institutions, potentially limiting generalizability of the study findings to similar institutions. Other institutions may also have a different cost structure and treatment preferences, which may greatly affect the cost of care. The small sample sizes could have an effect on
TABLE 3. Univariable analysis of cost drivers for SRS

\begin{tabular}{lcc}
\hline \multirow{2}{*}{ Variable } & \multicolumn{2}{c}{ Univariable } \\
\cline { 2 - 3 } & Standardized $\beta$ & $\mathrm{p}$ Value \\
\hline Age in yrs & -0.2 & 0.2 \\
\hline Male sex & 0.1 & 0.4 \\
\hline LOS in days & -0.002 & 0.99 \\
\hline Max tumor size in $\mathrm{cm}$ & 0.06 & 0.7 \\
\hline Serviceable hearing & -0.1 & 0.4 \\
\hline Koos grade & 0.07 & 0.6 \\
\hline Fundal fluid cap & 0.007 & 0.96 \\
\hline
\end{tabular}

Because none of the variables were $p<0.2$ on univariate analysis, multivariable analysis was not performed.

costs because the clinical heterogeneity of each patient has a larger effect on the costs for each group. In our study we limited our assessment of costs to those associated with the perioperative time frame and omitted additional costs, such as long-term follow-up and postoperative care costs (imaging, readmission, clinic visits) and the indirect socioeconomic costs of missed work days and disability. These factors make an overall cost-effectiveness comparison of open surgical and SRS cases challenging. Further studies would be necessary to better understand how cost considerations can inform the selection of patient treatment options.

\section{Conclusions}

Microsurgical resection and radiosurgery remain the most widely used interventions in the treatment of vestibular schwannomas. Because healthcare systems and programs are demanding greater attention to cost-effectiveness, we have undertaken this analysis to delineate the factors that drive the total costs of these two treatment options. When healthcare providers consider costs, they are serving the interests of their patients. ${ }^{19}$ Therefore, it is essential to develop protocols to improve resource utilization and reduce costs while maximizing patient care. Our goal was to identify areas in which measures to control costs can have the most effect. We found that facility utilization constitutes the major factor for total costs in both treatment modalities. Thus, strategies to improve cost for patients, including types of surgical units and other facility-use improvements, should be evaluated to improve cost-effective care for patients with vestibular schwannoma. For example, use of a surgical step-down unit for patients with low postoperative risk or streamlining discharge processing to avoid unnecessary days in the hospital could potentially reduce costs.

\section{Acknowledgments}

We thank Kristin Kraus, MSc, for her editorial assistance and Jacob Kresser for assistance with the VDO database.

\section{References}

1. Banerjee R, Moriarty JP, Foote RL, Pollock BE: Comparison of the surgical and follow-up costs associated with microsur- 
gical resection and stereotactic radiosurgery for vestibular schwannoma. J Neurosurg 108:1220-1224, 2008

2. Caruso JP, Moosa S, Fezeu F, Ramesh A, Sheehan JP: A cost comparative study of Gamma Knife radiosurgery versus open surgery for intracranial pathology. J Clin Neurosci 22:184-188, 2015

3. Chin LS, Ma L, DiBiase S: Radiation necrosis following gamma knife surgery: a case-controlled comparison of treatment parameters and long-term clinical follow up. J Neurosurg 94:899-904, 2001

4. Guan J, Karsy M, Bisson EF, Couldwell WT: Patient-level factors influencing hospital costs and short-term patientreported outcomes after transsphenoidal resection of sellar tumors. Neurosurgery [epub ahead of print], 2017

5. Karsy M, Brock AA, Guan J, Bisson EF, Couldwell WT: Assessment of cost drivers in transsphenoidal approaches for resection of pituitary tumors using the Value-Driven Outcome Database. World Neurosurg 105:818-823, 2017

6. Kawamoto K, Martin CJ, Williams K, Tu MC, Park CG, Hunter C, et al: Value Driven Outcomes (VDO): a pragmatic, modular, and extensible software framework for understanding and improving health care costs and outcomes. J Am Med Inform Assoc 22:223-235, 2015

7. Loeffler JS, Niemierko A, Chapman PH: Second tumors after radiosurgery: tip of the iceberg or a bump in the road? Neurosurgery 52:1436-1442, 2003

8. Myrseth E, Pedersen PH, Møller P, Lund-Johansen M: Treatment of vestibular schwannomas. Why, when and how? Acta Neurochir (Wien) 149:647-660, 2007

9. Oghalai JS, Buxbaum JL, Pitts LH, Jackler RK: The effect of age on acoustic neuroma surgery outcomes. Otol Neurotol 24:473-477, 2003

10. Reese JC, Karsy M, Twitchell S, Bisson EF: Analysis of anterior cervical discectomy and fusion healthcare costs via the value-driven outcomes tool. Neurosurgery [epub ahead of print], 2018

11. Schmidt RF, Boghani Z, Choudhry OJ, Eloy JA, Jyung RW, Liu JK: Incidental vestibular schwannomas: a review of prevalence, growth rate, and management challenges. Neurosurg Focus 33(3):E4, 2012

12. Schmitt WR, Carlson ML, Giannini C, Driscoll CL, Link MJ: Radiation-induced sarcoma in a large vestibular schwannoma following stereotactic radiosurgery: case report. Neurosurgery 68:E840-E846, 2011

13. Slattery WH, Schwartz MS, Fisher LM, Oppenheimer M: Acoustic neuroma surgical cost and outcome by hospital volume in California. Otolaryngol Head Neck Surg 130:726735, 2004

14. Sonig A, Khan IS, Wadhwa R, Thakur JD, Nanda A: The impact of comorbidities, regional trends, and hospital factors on discharge dispositions and hospital costs after acoustic neuroma microsurgery: a United States nationwide inpatient data sample study (2005-2009). Neurosurg Focus 33(3):E3, 2012

15. Stangerup SE, Tos M, Thomsen J, Caye-Thomasen P: True incidence of vestibular schwannoma? Neurosurgery 67:1335-1340, 2010
16. Twitchell S, Abou-Al-Shaar H, Reese J, Karsy M, Eli IM, Guan J, et al: Analysis of cerebrovascular aneurysm treatment cost: retrospective cohort comparison of clipping, coiling, and flow diversion. Neurosurg Focus 44(5):E3, 2018

17. Twitchell S, Karsy M, Reese J, Guan J, Couldwell WT, Dailey A, et al: Assessment of cost drivers and cost variation for lumbar interbody fusion procedures using the Value Driven Outcomes database. Neurosurg Focus 44(5):E10, 2018

18. van Roijen L, Nijs HG, Avezaat CJ, Karlsson G, Linquist C, Pauw KH, et al: Costs and effects of microsurgery versus radiosurgery in treating acoustic neuroma. Acta Neurochir (Wien) 139:942-948, 1997

19. Verma S, Anthony R, Tsai V, Taplin M, Rutka J: Evaluation of cost effectiveness for conservative and active management strategies for acoustic neuroma. Clin Otolaryngol 34:438446, 2009

20. Wellis G, Nagel R, Vollmar C, Steiger HJ: Direct costs of microsurgical management of radiosurgically amenable intracranial pathology in Germany: an analysis of meningiomas, acoustic neuromas, metastases and arteriovenous malformations of less than $3 \mathrm{~cm}$ in diameter. Acta Neurochir (Wien) 145:249-255, 2003

21. Whitmore RG, Urban C, Church E, Ruckenstein M, Stein SC, Lee JY: Decision analysis of treatment options for vestibular schwannoma. J Neurosurg 114:400-413, 2011

22. Wise SC, Carlson ML, Tveiten OV, Driscoll CL, Myrseth E, Lund-Johansen M, et al: Surgical salvage of recurrent vestibular schwannoma following prior stereotactic radiosurgery. Laryngoscope 126:2580-2586, 2016

23. Zygourakis CC, Oh T, Sun MZ, Barani I, Kahn JG, Parsa AT: Surgery is cost-effective treatment for young patients with vestibular schwannomas: decision tree modeling of surgery, radiation, and observation. Neurosurg Focus 37(5):E8, 2014

\section{Disclosures}

Dr. Jensen is a consultant for Medtronic.

\section{Author Contributions}

Conception and design: Couldwell, Abou-Al-Shaar, Karsy, Jensen. Acquisition of data: Abou-Al-Shaar, Azab, Karsy, Guan, Alzhrani, Gozal. Analysis and interpretation of data: Abou-Al-Shaar, Karsy. Drafting the article: Abou-Al-Shaar, Karsy. Critically revising the article: all authors. Reviewed submitted version of manuscript: Couldwell, Abou-Al-Shaar, Karsy. Approved the final version of the manuscript on behalf of all authors: Couldwell. Study supervision: Couldwell, Jensen.

\section{Correspondence}

William T. Couldwell: University of Utah, Salt Lake City, UT. neuropub@hsc.utah.edu. 